\title{
Caracterización de los usos de las poblaciones de palmas en las localidades de Angostura y Tutunendo, Chocó y sus efectos sobre la estructura de sus comunidades naturales
}

\author{
Characterización the uses of the populations of palms of locations Angostura \\ and Tutunendo, Chocó and its effects on the structure of them natural \\ communities
}

\author{
Giovanny Ramírez Moreno, MSc*
}

RESUMEN

La extracción que a menudo se realiza en tierras sin posesión reconocida, donde muchos individuos explotan el mismo producto al mismo tiempo, sumado a la falta de herramientas de cosecha y métodos adecuados que permitan utilizar las palmas sin talarlas, son sus principales amenazas en el Chocó. Estos motivos impulsaron al estudio de las comunidades de palmas de Angostura y Tutunendo, Chocó, de donde se derivó la caracterización de usos y posibles efectos provocados por los mismos, mediante la utilización de herramientas que combinaron información etnobotánica (in situ y ex situ), con levantamientos y análisis de estructura de las comunidades de palmas. De las 33 especies registradas, 28 reportan uso por los habitantes de la comunidad. Se identificaron siete categorías, siendo la más frecuente la artesanal (18 especies), seguida de construcción (17), elaboración de utensilios y herramientas (11), alimentación humana (10), medicinal y/o veterinaria (5), cultural (4) y venenosas con un solo reporte de uso. Los órganos más usados fueron tallo en 21 especies, hojas en 14 y frutos en seis. Datos estructurales permiten inferir efectos de uso de órganos en la estructura poblacional de las palmas, como se observas en Welfia regia, cuya estructura poblacional presenta más juveniles (50\%) y adultos (26\%) que plántulas (24\%), situación que se puede relacionar con la categoría de uso (construcción) y el órgano utilizado (tallos y hojas).

Palabras clave: Chocó; Comunidades; Estructura; Poblaciones; Palmas; Usos.

\section{ABSTRACT}

The extraction is often carried out on land without possessing recognized, where many individuals exploit the same product at the same time, coupled with the lack of harvesting tools and methods, allowing the palms to be used without being cleared, are the main threats of them in the Chocó. These reasons prompted the study of palm communities of these two locations, from which I derive this characterization of uses and potential impacts caused by them. Using tools that combined ethnobotanical information (in situ and ex situ), with surveys and analysis of community structure of palms. Of the 33 species recorded 28 species reported use by community residents. We identified seven categories, the scale was the mostcommon (18 species), followed by construction (17), development of tools and utensils (11), Food (10), medicinal and / or veterinary (5), cultural (4) and poisonous to a single report of use. The organs

* Investigador Principal Componente Ecosistémico, Instituto de Investigaciones Ambientales del Pacífico-IIAP. Grupo de Investigación en conocimiento, manejo y conservación de los ecosistemas del Chocó biogeográfico. Grupo de Investigación en Palmas Neotropicales, Universidad Nacional de Colombia, Bogotá, Colombia. e-mail:gramirez@iiap.org.co 
used were 21 species stems, leaves and fruits from 14 to 6. Structural data allow us to infer effects of use of organs in the population structure of the palms. As noted in Welfia regia, which presents more youthful population structure (50\%) and adults (26\%) than seedlings (24\%), a situation that may be related to the use category (construction) and the organ used (stems and leaves).

Keywords: Chocó; Communities; Structure; Populations; Palms; Uses.

\section{INTRODUCCIÓN}

La gran riqueza y la alta representatividad de las palmas en los bosques tropicales, han provocado la estrecha relación que tienen con el hombre, a tal punto que las palmas se consideran la tercera familia de plantas más útiles en el trópico, después de las gramíneas y las leguminosas (Johnson 1996), importancia que es más acentuada en las zonas rurales y selváticas, donde las palmas proveen al hombre de un sinnúmero de bienes para su uso y consumo (Galeano y Bernal 2005); además, como lo mencionó Balick (1982), las palmeras están profundamente involucradas en las creencias mágicas y místicas, y son elementos de rutina en el folclore regional o tribal. Esto también se evidencia claramente en la región del Pacífico, donde según Bernal y Galeano (1993) y García et al. (2002), son variados y numerosos los usos que la comunidad chocoana da a este valioso recurso, entre los que se pueden mencionar alimento, artesanías, fibra, aceites y/o grasas, palmitos, construcción, vivienda, medicina y vestido. Según Valois y Pino (2004) y Castro et al. (2004), la subregión central del Chocó no es ajena a la estrecha relación entre las palmas y el hombre, como lo muestra el hecho de que en cuatro comunidades negras del municipio de Quibdó, se reportaron las palmas como la familia más representativa dentro las categorías de uso.

Es lamentable que a pesar de la gran riqueza de palmas y su importancia para el hombre, estén expuestas a múltiples amenazas. Según Galeano y Bernal (2005), el panorama para las palmas en la región del Pacífico no es alentador, porque aunque aún se conservan extensas áreas de bosque, especialmente en la zona central, en esta región se encuentran siete especies en alguna categoría de amenaza, tres de ellas endémicas. Además de la pérdida de hábitats, dos de las principales amenazas según Borgtoft Pedersen y Balslev (1993) son : primero, la extracción, que a menudo se lleva a cabo en tierras sin posesión reconocida, lo que hace que muchos individuos exploten el mismo producto al mismo tiempo. Segundo, hacen falta herramientas de cosecha y métodos adecuados, mediante los cuales las palmas se utilicen sin talarlas. Estos motivos impulsaron el estudio de las comunidades de palmas de estas dos localidades.
El objetivo general es caracterizar los usos de las poblaciones de palmas en las localidades de Angostura y Tutunendo, Chocó y sus efectos sobre la estructura de sus comunidades naturales.

\section{METODOLOGÍA}

Área de estudio. El presente estudio se realizó en dos estaciones ambientales del Instituto de Investigaciones Ambientales del Pacífico, donde se centran los esfuerzos de investigación del Instituto, una ubicada en la localidad de Tutunendo y la otra en la localidad de Angostura. Estas dos estaciones cubren áreas que representan una parte importante del departamento del Chocó, una de las áreas más biodiversas del continente. El departamento del Chocó, situado en la esquina noroccidental de Suramérica, entre $4^{\circ}-8^{\circ} \mathrm{N}$ y $76^{\circ}-78^{\circ}$ $\mathrm{W}$, es la única región de Suramérica con costa sobre dos océanos, el Atlántico al norte y el Pacífico al occidente. Tiene una extensión de $47.840 \mathrm{~km}^{2}$ y ocupa por ello el cuarto lugar entre los departamentos de Colombia. Cuenta con vías fluviales importantes como los ríos Atrato, San Juan y Baudó, entre otros (Eslava 1993). Para el departamento se han reconocido seis zonas de vida: bosque pluvial-premontano (bp-PM), bosque muy húmedo-tropical (bmh-T), bosque muy húmedopremontano (bmh-PM), bosque húmedo-tropical (bh-T), bosque pluvial-montano bajo (bp-MB) y bosque pluvial-montano (bp-M) (Holdridge 1996); según datos registrados en la Estación Meteorológica de la Universidad Tecnológica del Chocó, en el departamento se presenta una temperatura promedio anual de $25^{\circ} \mathrm{C}$ y una humedad relativa de más del $90 \%$. Según la clasificación de Forero y Gentry (1989), existen ocho regiones fitogeográficas, dos de ellas, la selva pluvial central y la subregión del San Juan, correspondientes a las zonas de estudio (la selva pluvial central soporta la más alta precipitación de la región, con cerca de $11.000 \mathrm{~mm}$ para Tutunendo, mientras que la región del Bajo San Juan está en la franja transicional de bosque muy húmedo a bosque húmedo, influenciada por la cercanía al piedemonte cordillerano) (Figura 1).

Estación ambiental Tutunendo. Ubicada en el corregimiento de Tutunendo a los $05^{\circ} 44^{\prime} 41.7^{\prime \prime} \mathrm{N}-76^{\circ} 32^{\prime} 24.2^{\prime \prime} \mathrm{W}$ y $50 \mathrm{msnm}$, pertenee a la subregión fitogeográfica de la selva pluvial central, denominada así, por su localización en el borde de la planicie central del Chocó, donde el inicio de la influencia de la cordillera Occidental y la fuerte incidencia de los factores climáticos (precipitación) sobre la vegetación, modelan la estructura y la composición del bosque (Forero y Gentry 1989).

La estación ambiental de Tutunendo está conformada en su totalidad por un antiguo terreno familiar de 75 ha aproximadamente, adjudicado por comodato desde 1997 al IIAP; en estas tierras, por mucho tiempo, se combinaron actividades 


\section{Bioetnia Volumen 8 No 1 (enero-junio), 2011}

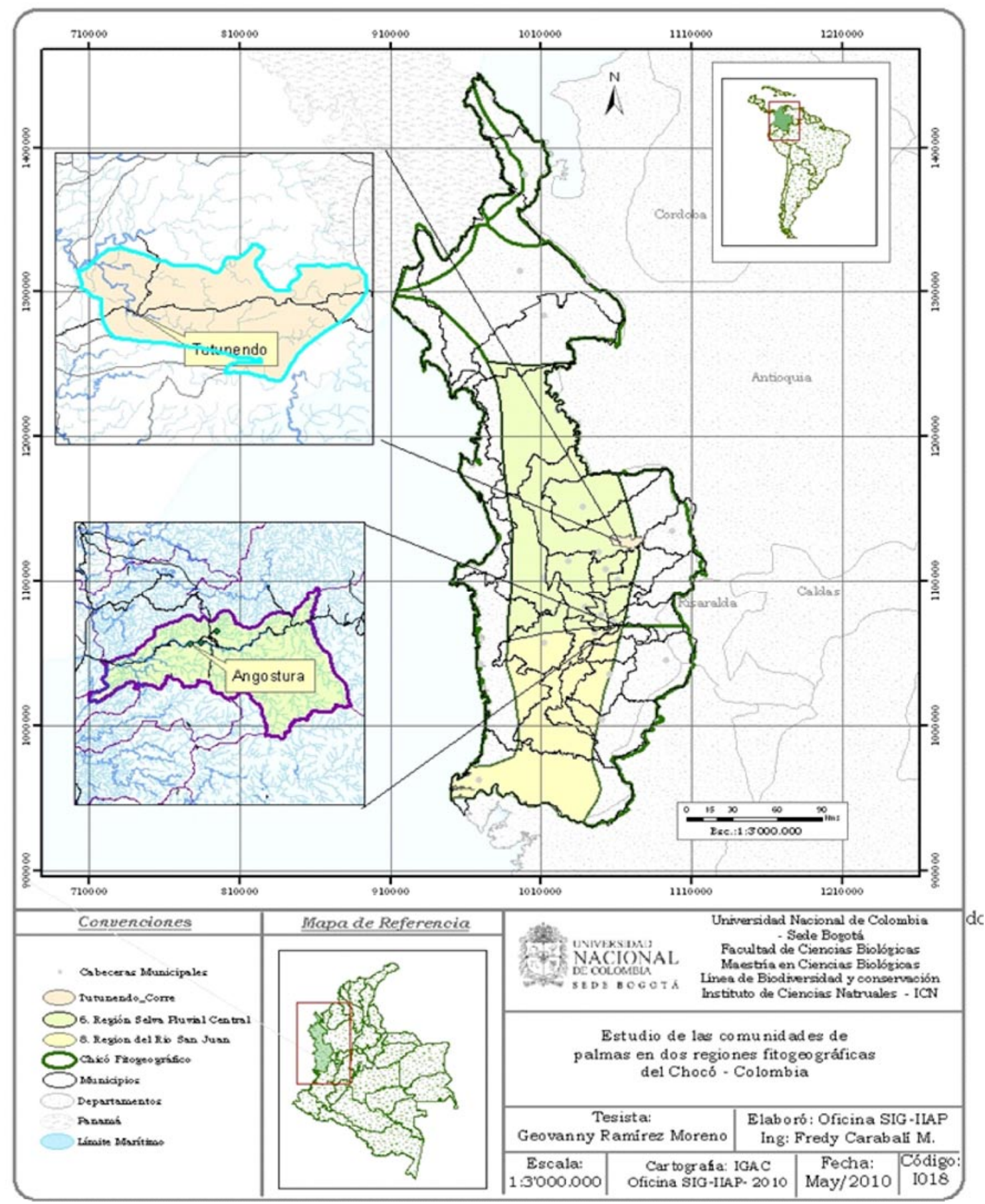

Figura 1. Ubicación de las localidades de estudio.

como la extracción selectiva de especies forestales, los cultivos de plátano y piña, y durante varios años en la década de los 80 , se introdujo, por un período de tiempo corto y a baja escala, la ganadería en pequeños parches.

Estación ambiental Alto San Juan (Angostura). Se en- cuentra ubicada en la parte alta del río San Juan, municipio de Tadó, corregimientos de Angostura y Playa de Oro; pertenece a la subregión fitogeográfica del San Juan, formada por el territorio ubicado a lo largo de la cuenca del río San Juan y se caracteriza por sus múltiples variaciones paisajísticas (plani- 
cies inundables, bosques de tierra firme y zonas de transición) (Forero y Gentry 1989).

El área total de la reserva es de 28 ha, pero el área de influencia, donde se establecieron las unidades de muestreo es de unas 110 ha. Esta zona ha sido fuertemente transformada por la actividad minera, que ha convertido grandes extensiones de bosque en sitios desolados sin cobertura boscosa; esta situación llevó al IIAP y a la Asociación Campesina del Alto Río San Juan (ASOCASAN) en el año 2003, a la creación de la estación ambiental, con el fin de recuperar, conocer y conservar los territorios colectivos de la zona (Figura 1).

\section{MÉTODOS}

Concertación con la comunidad. Se realizó esta etapa con varios propósitos; en primer lugar, informar a los habitantes de cada localidad de estudio sobre las pretensiones del proyecto y los resultados esperados, la metodología propuesta para desarrollarlo y los impactos que eventualmente se generarían con la aplicación de la información aportada; en segundo lugar, lograr acuerdos en cuanto a los mecanismos de participación de la comunidad, definir los sitios precisos de muestreo, y las rutas y costos necesarios para su acceso. Para tal efecto, se canalizaron los esfuerzos a través de los representantes legales del Consejo Comunitario de Tutunendo (COCMACIA) y de Angostura (Alto San Juan-ASOCASAN). Una vez identificadas las comunidades en cuyo territorio se adelantaría el proyecto, se realizó una convocatoria amplia a los miembros del Consejo Comunitario para llevar a cabo los talleres de concertación, donde se lograron los acuerdos previstos y se dispuso de los mecanismos para enfrentar la fase de campo.

Usos de las palmas por parte de los pobladores asentados en la zona de influencia del proyecto. En cada localidad se registró la información suministrada por cinco guías de campo y tres sabios tradicionales (16 personas), todos hombres mayores de 45 años, nativos de las localidades, reconocidos por los habitantes y consejos comunitarios como conocedores de los bosques. La información etnobotánica se obtuvo directamente en campo durante el trabajo en los transectos. Las preguntas acerca del uso de palmas fueron abiertas, dejando que los entrevistados mencionaran las especies que conocían, los nombres comunes, las especies usadas y la parte de la palma aprovechada. Todos los usos reportados se incluyeron en ocho categorías de uso: alimenticio, construcción, aceites, artesanal, utensilios de uso doméstico, medicinal, ceremonial y otros (que incluye a aquellos tipos de uso que no se pueden incluir en las categorías anteriores).

Para la compilación de la información anterior, se construyó una base de datos en Excel 2007, que incluyó información de los formularios de campo, como la posición geográfica del lugar de colección, los caracteres morfológicos, algunos aspectos ecológicos de los individuos colectados (como exposición a la luz, formas de crecimiento, estado fenológico, abundancia, frecuencia, preferencias de hábitats) y usos locales.

Para la determinación de la estructura de la comunidad de palmas, se cuantificaron los siguientes parámetros: densidad (D): Número de individuos de una especie/Número total de individuos; densidadrelativa(DR): D de una especie/Sumatoria de todas las densidades de todas las especies x 100; frecuencia (F): Número de transectos en los que se encuentra la especie/Número de transectos totales; frecuencia relativa (FR): F de la especie/Sumatoria de todas las frecuencias de todas las especies por 100 (Rangel y Velásquez 1997) y para analizar la importancia ecológica de cada una de las especies de palmas se obtuvo el índice de valor de importancia (IVI), que está dado por la sumatoria de los valores relativos de la densidad, la frecuencia y la dominancia relativa, que se expresa usualmente en términos de área basal (Villareal et al. 2004). Sin embargo, en este estudio el IVI para cada especie se obtuvo solo con la sumatoria de la densidad relativa y la frecuencia relativa.

\section{RESULTADOSYDISCUSIÓN}

Usos y formas de usos de las palmas en las localidades de Tutunendo y Angostura. La información etnobotánica muestra que de las 33 especies registradas en las localidades de estudio, 28 especies presentan reportes de uso por los habitantes de la comunidad. Se identificaron siete categorías, siendo la de mayor frecuencia la de uso artesanal (18 especies), seguida de construcción (17), elaboración de utensilios y herramientas (11), alimentación humana (10), medicinal y/o veterinaria (5), cultural (4) y venenosas con un solo reporte de uso (Tabla 1).

Se tuvo registro de uso tanto de los órganos reproductivos, como de los vegetativos de las palmas: raíz, tallo, hoja, bráctea peduncular, fruto y semilla. Los órganos más utilizados fueron el tallo en 21 especies, seguido de las hojas en 14 y los frutos en seis; en menor proporción (dos) se usan las semillas, brácteas y raíces, lo que se relaciona con el hecho de que las categorías de uso más frecuentes requieren del uso sobre todo del tallo y las hojas. En 35 de las combinaciones obtenidas entre categorías de uso y órgano utilizado, se presenta el tallo (35), seguido de las hojas (20), frutos (10), semillas (3), brácteas y raíces (2) (Figura 2).

La especie con mayor número de usos es Oenocarpus bataua (milpesos), ya que se reporta en seis de las siete categorías, con el aprovechamiento de cuatro de sus órganos (tallo, hojas, brácteas y frutos). En segundo lugar se encuentra Oenocarpus minor (donpedrito) (cinco categorías), seguida de Desmoncus cirrhifer y Euterpe precatoria (cuatro 


\section{Bioetnia Volumen 8 No 1 (enero-junio), 2011}

Tabla 1

Categorías de uso de las palmas de Tutunendo y Angostura

\begin{tabular}{|c|c|c|c|c|c|c|c|c|}
\hline Especies & Nombre vulgar & ALI & ART & CON & CUL & MED & $\mathbf{v}$ & U Y H \\
\hline Asterogyne martiana & Cuchilleja & & & $\mathrm{H}$ & & & & \\
\hline Attalea alleni & Táparo & $\mathrm{S}$ & B & & $\mathrm{H}$ & & & \\
\hline Bactris barronis & Chontadurillo & $\mathrm{F}$ & & $\mathrm{T}$ & & & & \\
\hline Bactris brongniartii & Chacarrá & $\mathrm{F}$ & & & & & & $\mathrm{T}$ \\
\hline Bactris coloniata & Chacarrá & $\mathrm{F}$ & $\mathrm{T}$ & & & & & $\mathrm{T}$ \\
\hline Bactris hondurensis & Chacarracito & & $\mathrm{T}$ & & & & & \\
\hline Bactris maraja & Chacarrá & $\mathrm{F}$ & & $\mathrm{T}$ & & & & \\
\hline Desmoncus cirrhifer & Matamba & & $\mathrm{T}$ & & $\mathrm{T}$ & & & $\mathrm{T}$ \\
\hline Euterpe precatoria & Naidí & $\mathrm{F}, \mathrm{H}$ & $\mathrm{T}$ & $\mathrm{T}$ & & & & $\mathrm{T}$ \\
\hline Geonoma calyptrogynoidea & No conocido & & & $\mathrm{H}$ & & & & \\
\hline Geonoma cuneata & Rabiorcado & & $\mathrm{H}$ & $\mathrm{H}$ & & & & \\
\hline Geonoma deversa & Palmiche & & $\mathrm{T}$ & & & & & $\mathrm{T}$ \\
\hline Geonoma divisa & Palmilla & & $\mathrm{T}$ & & & & & $\mathrm{T}$ \\
\hline Geonoma stricta & Palmiche & & $\mathrm{T}$ & & & & & $\mathrm{T}$ \\
\hline Hyosphate elegans & No conocido & & $\mathrm{T}$ & & & & & $\mathrm{T}$ \\
\hline Iriartea deltoidea & Barrigona & & $\mathrm{T}$ & $\mathrm{T}$ & & & & \\
\hline Mauritiella macroclada & Quitasol & & $\mathrm{T}$ & $\mathrm{T}, \mathrm{H}$ & & & & \\
\hline Oenocarpus bataua & Milpesos & $\mathrm{F}$ & B & $\mathrm{H}, \mathrm{T}$ & $\mathrm{H}$ & & & $\mathrm{F}$ \\
\hline Oenocarpus minor & Don pedrito, Murrapo & $\mathrm{F}, \mathrm{T}$ & S & $\mathrm{T}, \mathrm{H}$ & & & & $\mathrm{F}$ \\
\hline Pholydostachis dactyloides & Rabo de zorro & & $\mathrm{H}$ & & $\mathrm{H}$ & & & \\
\hline Pholydostachis pulcra & Rabo de zorro & & $\mathrm{H}$ & & $\mathrm{H}$ & & & \\
\hline Socratea exorrhiza & Zancona & & $\mathrm{R}, \mathrm{S}$ & $\mathrm{T}$ & & & & $\mathrm{R}$ \\
\hline Synechanthus warcewizcianus & No conocido & & $\mathrm{T}$ & & & $\mathrm{H}$ & & \\
\hline Welfia regia & Amargo & & $\mathrm{H}$ & $\mathrm{H}, \mathrm{T}$ & & & $\mathrm{H}$ & \\
\hline Wettinia aequalis & Sapa & & & $\mathrm{T}$ & & & & \\
\hline Wettinia quinaria & Memé & & S & $\mathrm{T}$ & & $\mathrm{H}$ & & \\
\hline Wettinia radiata & No conocido & & & $\mathrm{T}$ & & $\mathrm{H}$ & & \\
\hline
\end{tabular}

alimento (ALI), artesanal (ART), construcción (CON), cultural (CUL), medicinal (MED), venenosa (V), utensilios y herramientas (UYH). Parte utilizada, raíz $(R)$, tallo $(T)$, hoja $(H)$, fruto $(F)$, bráctea $(B)$, semilla $(S)$.

categorías); algunas como Attalea alleni, Bactris coloniata, Socratea exorrhiza, Welfia regia y Wettinia quinaria con tres categorías, prevaleciendo la utilización de tallo y hojas; las demás especies se distribuyen entre una y dos categorías de uso. Sin embargo, el panorama de aprovechamiento en ambas localidades muestra que especies con un número menor de usos registrados (dos o tres categorías y dos órganos), como es el caso de Iriartea deltoidea (barrigona) y Welfia regia (amargo), son muy apreciadas por los pobladores y por ello se emplean con mayor frecuencia en las labores cotidianas (Tabla 2).

Las siete categorías de uso asignadas a 29 de las 33 especies encontradas, permitieron demostrar la variada utilización de las palmas por parte de las poblaciones locales. Esta situación se relaciona estrechamente con la riqueza de especies, lo que coincide con las afirmaciones de Macía et al. (2001), quien relaciona la diversidad de usos con la diversidad de especies. Autores como García et al. (2002) afirman que la gran oferta natural que presenta este recurso, influye en el uso de las palmas en el Chocó, situación que se pudo corroborar en el presente estudio para especies como Wettinia quinaria, Socratea exorrhiza, Iriartea deltoidea, Welfia regia, Oenocarpus bataua, Oenocarpus minor, Euterpe precatoria, Geonoma cuneata y Geonoma calyptrogynoidea, que repre- 
Usos de las palmas y su efecto en la estructura de la comunidad. G. Ramírez

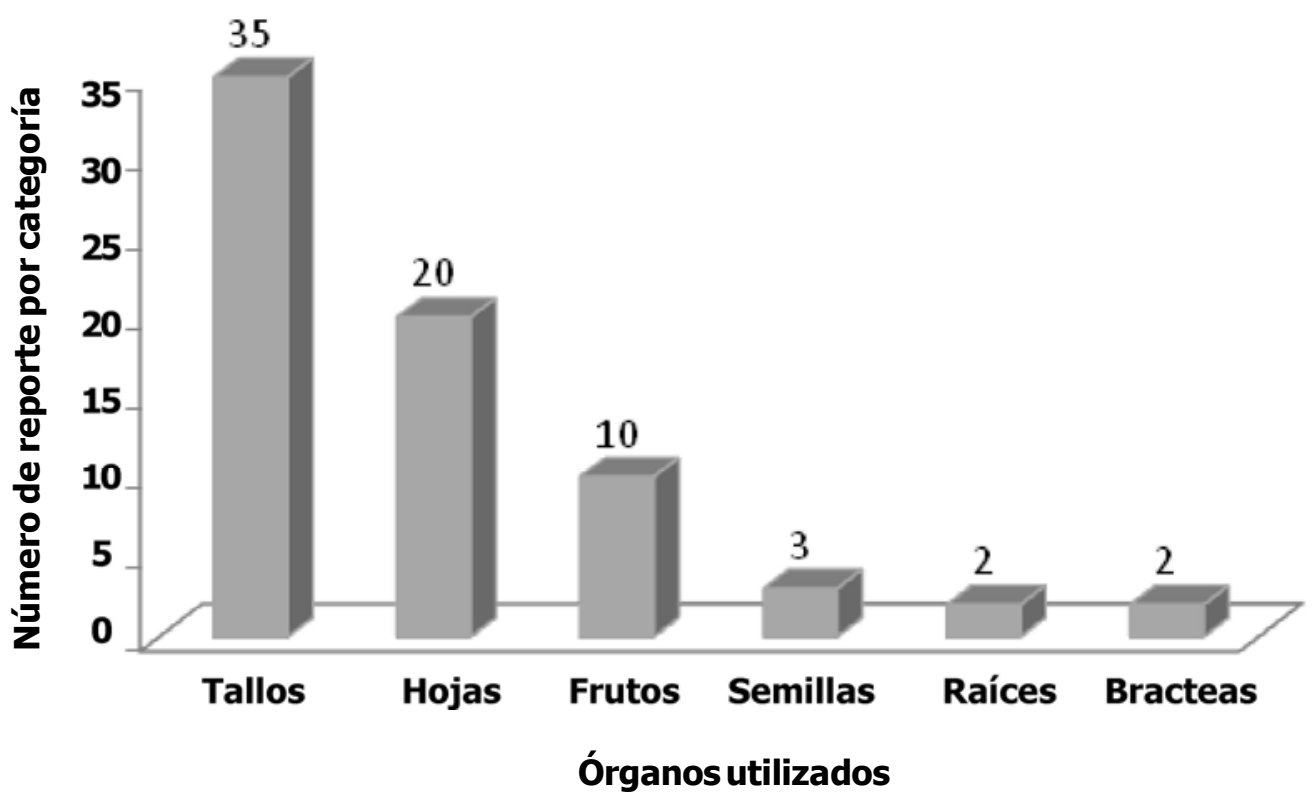

Figura 2. Órganos de la palma utilizados con mayor frecuencia.

sentaron más del $60 \%$ de los individuos de las comunidades de palmas en ambas localidades y que reportan también mayor número de usos. La accesibilidad al recurso también es un factor determinante en el grado de utilización del mismo (Benz et al. 2000; Macía et al. 2001; Byg y Balslev 2004), de allí que la cercanía de asentamientos humanos a las localidades de estudio, influyera en la cantidad de reportes de uso dados por los pobladores.

Los datos estructurales permiten inferir el efecto de la utilización de los órganos en la estructura de las poblaciones de palmas; es decir, que el uso de las especies tiene un impacto negativo que se evidencia en la estructura de las poblaciones usadas. Tal es el caso de Welfia regia, cuya estructura de la población presenta más juveniles $(50 \%)$ y adultos $(26 \%)$, que plántulas $(24 \%)$, situación que se relaciona con la categoría de uso (construcción) y el órgano utilizado (tallos y hojas), pues es frecuente que las palmas se derriben para la cosecha, lo que explicaría la deficiencia en el reclutamiento. Para las especies $O$. minor y $O$. bataua, se observó una estructura poblacional que evidencia una situación aún más dramática, con más adultos (68\% y 58\%, respectivamente) y juveniles (29\% y $40 \%$, respectivamente) que plántulas $(3 \%$ y $2 \%$, respectivamente), lo que se puede explicar porque la categoría de uso alimenticia requiere del aprovechamiento total de los frutos maduros, lo que podría incidir directamente sobre un muy deficiente reclutamiento de estas especies en las zonas estudiadas. De hecho, es bien conocido que los frutos de estas dos especies están entre los más apetecidos en toda la región del Chocó y que infortunadamente, en la mayoría de los casos, las palmas se derriban para cosechar los frutos.

\section{ESTRUCTURA DE LAS COMUNIDADES DE PAL- MAS DE LAS LOCALIDADES DE ESTUDIO}

Distribución por clases de tamaño de las comunidades de palmas en Tutunendo. Los datos del conteo de todos los individuos contenidos en los transectos, permitieron distribuir los 3818 individuos de la comunidad de palmas en los estados de desarrollo plántulas, juveniles y adultos de la siguiente manera: el mayor porcentaje de individuos se encontró en el estado juvenil (40\%), seguido de los adultos (32\%) y por último las plántulas $(28 \%)$ (Figura 3$)$.

Importancia ecológica de las especies. En la Tabla 3 se presentan los valores de importancia y sus respectivos componentes para cada una de las especies y sus respectivos estados de desarrollo (plántulas, juveniles y adultos) encontradas en Tutunendo. Más del $60 \%$ de la densidad de los individuos de palmas encontrados en la localidad de Tutunendo estuvo representada por sólo tres especies: Wettinia quinaria $(0,36)$, Welfia regia $(0,14)$ y Socratea exorrhiza $(0,12)$. La frecuencia de especies por transectos para la comunidad de palmas de la localidad de Tutunendo fue alta, encontrándose que 13 especies (45\%) de las 29, crecen en más de la mitad de los transectos; las especies más frecuentes fueron $W$. quinaria, $W$. regia e Iriartea deltoidea, que se hallaron en todos los transectos. Es importante mencionar que se observó un grupo de siete especies para las cuales la frecuencia y la abundancia fueron entre uno y cinco sitios, y entre 1 y 42 individuos. Las especies con mayor índice de valor de importancia fueron $W$. quinaria, $W$. regia, $S$. exorrhiza, I. deltoidea y G. cuneata; estas especies no solo 
Tabla 2

Especies con número de categorías de usos reportados

\begin{tabular}{ll}
\hline Especies & Categorías \\
\hline Oenocarpus bataua & 6 \\
Oenocarpus minor & 5 \\
Desmoncus cirrhifer & 4 \\
Euterpe precatoria & 4 \\
Attalea alleni & 3 \\
Bactris coloniata & 3 \\
Socratea exorrhiza & 3 \\
Welfia regia & 3 \\
Wettinia quinaria & 3 \\
Bactris barronis & 2 \\
Bactris brongniartii & 2 \\
Bactrismaraja & 2 \\
Geonoma cuneata & 2 \\
Geonoma deversa & 2 \\
Geonoma divisa & 2 \\
Geonoma stricta & 2 \\
Hyosphate elegans & 2 \\
Iriartea deltoidea & 2 \\
Mauritiella macroclada & 2 \\
Pholydostachis dactyloides & 2 \\
Pholydostachis pulcra & 2 \\
Synechanthus warcewizcianus & 2 \\
Wettinia radiata & 2 \\
Asterogyne martiana & 1 \\
Bactris hondurensis & 1 \\
Geonoma calyptrogynoidea & 2 \\
Wettinia equalis & \\
\hline & \\
\hline
\end{tabular}

son las más frecuentes, apareciendo en casi todos los transectos, sino que también dominan por sus altas densidades; el 50\% del total del IVI se concentró en estas cinco especies, que representan el 17\% del total de especies (Tabla 3).

Es importante destacar que al analizar de forma separada los datos correspondientes solo a individuos adultos de la localidad de Tutunendo, se observó que se mantiene la importancia de las especies conservando el mismo orden que se presenta en el análisis conjunto de las palmas en todos sus estados de desarrollo, lo que demuestra que las especies $W$. quinaria, W. regia, S. exorrhiza, I. deltoidea y G. cuneata están entre las especies dominantes en estos bosques (Tabla 4).

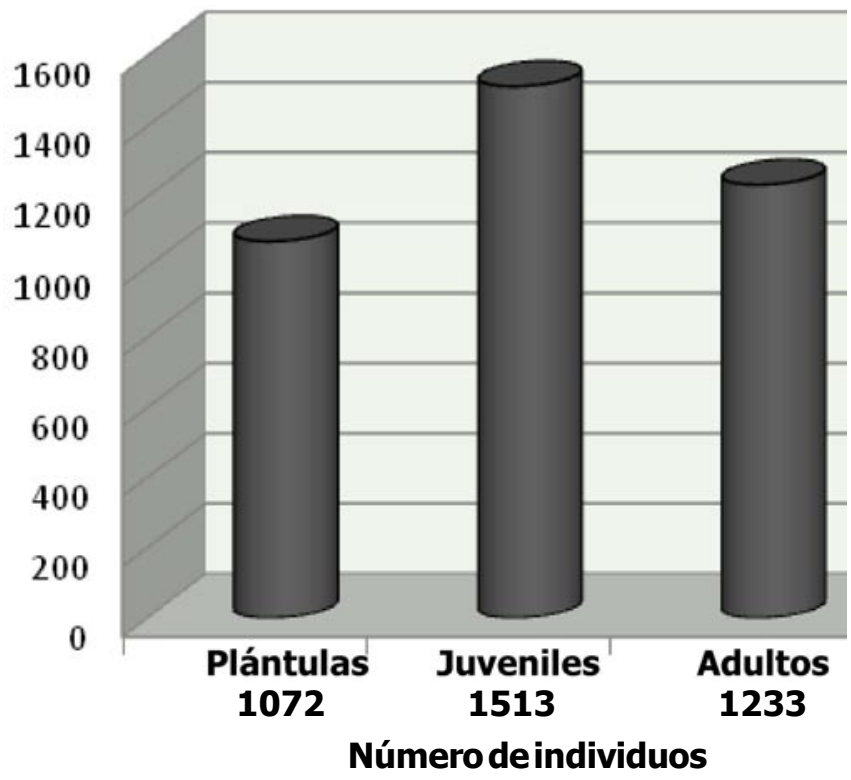

Figura 3. Distribución por clases de tamaño de las comunidades de palmas en 1,5 ha en la localidad de Tutunendo, Chocó, Colombia.

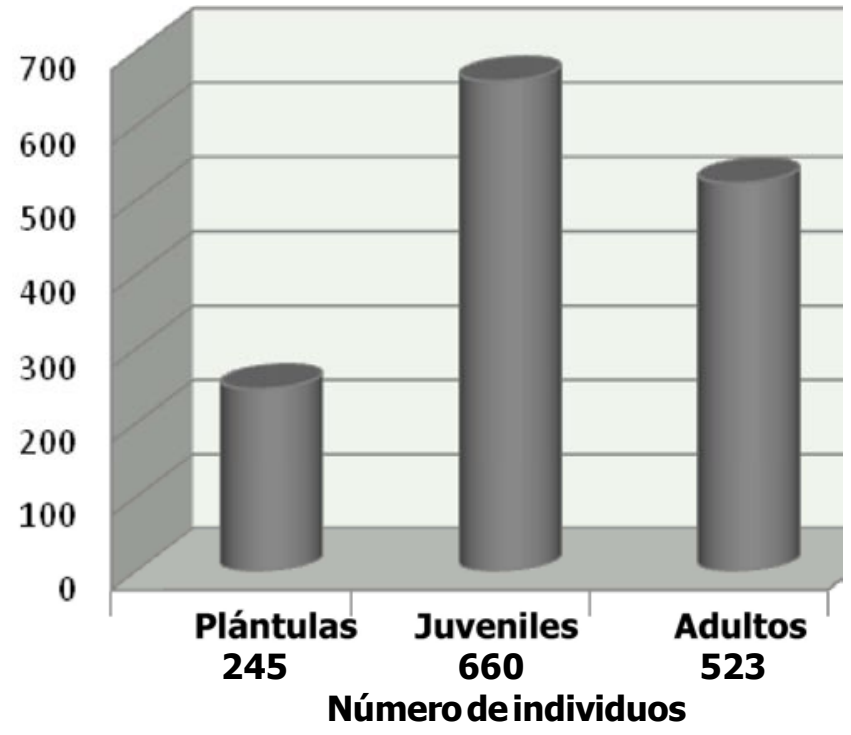

Figura 4. Distribución por clases de tamaño de la comunidad de palmas de Angostura.Importancia ecológica de las especies.

Distribución de las clases de tamaño en la comunidad de palmas de la localidad de Angostura. La distribución de los individuos según la clase de tamaño, mostró la dominancia de los juveniles con el $46 \%$, seguido del estado adulto con $37 \%$ y por último plántulas con $17 \%$ de los individuos de la localidad(Figura 4). 
Tabla 3

Estructura de la comunidad de palmas (plántulas, juveniles y adultos) encontradas en la localidad de Tutunendo, Quibdó, Chocó, Colombia

\begin{tabular}{|c|c|c|c|c|c|c|c|}
\hline Especies & NI & D & DR & $\mathbf{P}$ & $\mathbf{F}$ & $\mathbf{F R}$ & IVI \\
\hline Wettinia quinaria & 1387 & 0,36 & 0,3633 & 14 & 1,00 & 0,06763 & 0,43 \\
\hline Welfia regia & 544 & 0,14 & 0,1425 & 14 & 1,00 & 0,06763 & 0,21 \\
\hline Socratea exorhiza & 451 & 0,12 & 0,1181 & 12 & 0,86 & 0,05797 & 0,18 \\
\hline Iriartea deltoidea & 115 & 0,03 & 0,0301 & 14 & 1,00 & 0,06763 & 0,10 \\
\hline Geonoma cuneata & 116 & 0,03 & 0,0304 & 13 & 0,93 & 0,06280 & 0,09 \\
\hline Pholidostachys dactyloides & 159 & 0,04 & 0,0416 & 9 & 0,64 & 0,04348 & 0,09 \\
\hline Bactris coloniata & 141 & 0,04 & 0,0369 & 9 & 0,64 & 0,04348 & 0,08 \\
\hline Geonoma deversa & 121 & 0,03 & 0,0317 & 11 & 0,79 & 0,05314 & 0,08 \\
\hline Geonoma divisa & 98 & 0,03 & 0,0257 & 12 & 0,86 & 0,05797 & 0,08 \\
\hline Bactris hondurensis & 63 & 0,02 & 0,0165 & 11 & 0,79 & 0,05314 & 0,07 \\
\hline Oenocarpus bataua & 73 & 0,02 & 0,0191 & 10 & 0,71 & 0,04831 & 0,07 \\
\hline Oenocarpus minor & 80 & 0,02 & 0,0210 & 11 & 0,79 & 0,05314 & 0,07 \\
\hline Euterpe precatoria & 125 & 0,03 & 0,0327 & 6 & 0,43 & 0,02899 & 0,06 \\
\hline Geonoma calyptrogynoidea & 60 & 0,02 & 0,0157 & 10 & 0,71 & 0,04831 & 0,06 \\
\hline Bactris brongniartii & 49 & 0,01 & 0,0128 & 8 & 0,57 & 0,03865 & 0,05 \\
\hline Geonoma stricta & 43 & 0,01 & 0,0113 & 7 & 0,50 & 0,03382 & 0,05 \\
\hline Asterogyne martiana & 37 & 0,01 & 0,0097 & 9 & 0,64 & 0,04348 & 0,05 \\
\hline Pholidostachys pulcra & 42 & 0,01 & 0,0110 & 5 & 0,36 & 0,02415 & 0,04 \\
\hline Attalea alleni & 15 & 0,00 & 0,0039 & 5 & 0,36 & 0,02415 & 0,03 \\
\hline Wettinia radiata & 19 & 0,00 & 0,0050 & 3 & 0,21 & 0,01449 & 0,02 \\
\hline Camaedorea deneversiana & 12 & 0,00 & 0,0031 & 3 & 0,21 & 0,01449 & 0,02 \\
\hline Aiphanes acaulis & 23 & 0,01 & 0,0060 & 3 & 0,21 & 0,01449 & 0,02 \\
\hline Wettinia equalis & 4 & 0,0010 & 0,0010 & 1 & 0,07 & 0,00483 & 0,01 \\
\hline Bactris barronis & 8 & 0,00 & 0,0021 & 1 & 0,07 & 0,00483 & 0,01 \\
\hline Desmoncus cirrhifer & 1 & 0,00 & 0,0003 & 1 & 0,07 & 0,00483 & 0,01 \\
\hline Hyosphate elegans & 6 & 0,00 & 0,0016 & 1 & 0,07 & 0,00483 & 0,01 \\
\hline Mauritiela macroclada & 9 & 0,00 & 0,0024 & 1 & 0,07 & 0,00483 & 0,01 \\
\hline Prestoea & 4 & 0,00 & 0,0010 & 1 & 0,07 & 0,00483 & 0,01 \\
\hline Synechantus warcewizcianus & 13 & 0,00 & 0,0034 & 2 & 0,14 & 0,00966 & 0,01 \\
\hline Total & 3818 & 1 & 1 & 207 & 14,79 & 1,00 & 2,00 \\
\hline
\end{tabular}

$\mathrm{NI}=$ Número de individuos, $\mathrm{D}=$ Densidad, $\mathrm{DR}=$ Densidad relativa, $\mathrm{P}=$ Presencia, F: Frecuencia, FR: Frecuencia relativa,

IVI: Índice de valor de importancia

Los valores de importancia ecológica de todas las especies halladas en Angostura se muestran en la Tabla 6. W. quinaria $(0,21), W$. regia $(0,15)$ y G. cuneata $(0,08)$, con más del $44 \%$ de la densidad relativa, afectaron fuertemente parámetros estructurales como la frecuencia de especies, encontrando 11 (48\%) de ellas que se repiten en menos de siete transectos; $10(43 \%)$ se repiten de siete hasta nueve transectos y las más frecuentes, $W$. quinaria y $W$. regia, aparecen en todos los transectos; el efecto de las altas densidades y frecuencias de $W$. quinaria, $W$. regia, y $G$. cuneata, las convirtieron en las especies con mayor índice de valor de importancia-IVI de $0,298,0,231$ y 0,142 , respectivamente; es importante mencionar que en estas se concentró el $67 \%$ del IVI de la comunidad de palmas de la localidad de Angostura (Tabla 5).

Los datos correspondientes a los individuos adultos de 
Bioetnia Volumen 8 No 1 (enero-junio), 2011

Tabla 4

Estructura de la comunidad de palmas (sólo adultos) encontradas en la localidad de Tutunendo, Quibdó, Chocó, Colombia

\begin{tabular}{|c|c|c|c|c|c|c|c|}
\hline Especies & NI & D & DR & $\mathbf{P}$ & $\mathbf{F}$ & $\mathbf{F R}$ & IVI \\
\hline Wettinia quinaria & 359 & 0,29116 & 0,29116 & 14 & 1 & 0,081871345 & 0,3730311 \\
\hline Welfia regia & 154 & 0,124899 & 0,124899 & 12 & 0,857 & 0,070175439 & 0,1950741 \\
\hline Socratea exorrhiza & 164 & 0,133009 & 0,133009 & 8 & 0,571 & 0,046783626 & 0,1797925 \\
\hline Iriartea deltoidea & 61 & 0,049473 & 0,049473 & 13 & 0,929 & 0,076023392 & 0,1254962 \\
\hline Geonoma deversa & 48 & 0,038929 & 0,038929 & 13 & 0,929 & 0,076023392 & 0,1149528 \\
\hline Oenocarpus minor & 49 & 0,03974 & 0,03974 & 11 & 0,786 & 0,064327485 & 0,104068 \\
\hline Geonoma divisa & 39 & 0,03163 & 0,03163 & 11 & 0,786 & 0,064327485 & 0,0959577 \\
\hline Pholidostachys dactyloides & 49 & 0,03974 & 0,03974 & 7 & 0,5 & 0,040935673 & 0,0806761 \\
\hline Geonoma cuneata & 31 & 0,025142 & 0,025142 & 9 & 0,643 & 0,052631579 & 0,0777735 \\
\hline Oenocarpus bataua & 38 & 0,030819 & 0,030819 & 8 & 0,571 & 0,046783626 & 0,0776028 \\
\hline Geonoma calyptrogynoidea & 28 & 0,022709 & 0,022709 & 8 & 0,571 & 0,046783626 & 0,0694925 \\
\hline Bactris coloniata & 34 & 0,027575 & 0,027575 & 7 & 0,5 & 0,040935673 & 0,0685107 \\
\hline Asterogyne martiana & 18 & 0,014599 & 0,014599 & 8 & 0,571 & 0,046783626 & 0,0613822 \\
\hline Euterpe precatoria & 32 & 0,025953 & 0,025953 & 5 & 0,357 & 0,029239766 & 0,0551927 \\
\hline Bactris hondurensis & 15 & 0,012165 & 0,012165 & 7 & 0,5 & 0,040935673 & 0,0531011 \\
\hline Bactris brongniartii & 16 & 0,012976 & 0,012976 & 6 & 0,429 & 0,035087719 & 0,0480642 \\
\hline Geonoma stricta & 22 & 0,017843 & 0,017843 & 5 & 0,357 & 0,029239766 & 0,0470824 \\
\hline Pholidostachys pulcra & 30 & 0,024331 & 0,024331 & 3 & 0,214 & 0,01754386 & 0,0418748 \\
\hline Wettinia radiata & 7 & 0,005677 & 0,005677 & 3 & 0,214 & 0,01754386 & 0,0232211 \\
\hline Aiphanes acaulis & 9 & 0,007299 & 0,007299 & 2 & 0,143 & 0,011695906 & 0,0189952 \\
\hline Chamaedorea deneversiana & 7 & 0,005677 & 0,005677 & 2 & 0,143 & 0,011695906 & 0,0173731 \\
\hline Synechanthus warcewizcianus & 4 & 0,003244 & 0,003244 & 2 & 0,143 & 0,011695906 & 0,01494 \\
\hline Mauritiella macroclada & 5 & 0,004055 & 0,004055 & 1 & 0,071 & 0,005847953 & 0,0099031 \\
\hline Wettinia equalis & 4 & 0,003244 & 0,003244 & 1 & 0,071 & 0,005847953 & 0,0090921 \\
\hline Hyosphate elegans & 4 & 0,003244 & 0,003244 & 1 & 0,071 & 0,005847953 & 0,0090921 \\
\hline Bactris barronis & 2 & 0,001622 & 0,001622 & 1 & 0,071 & 0,005847953 & 0,00747 \\
\hline Attalea alleni & 2 & 0,001622 & 0,001622 & 1 & 0,071 & 0,005847953 & 0,00747 \\
\hline Prestoea ensiformis & 1 & 0,000811 & 0,000811 & 1 & 0,071 & 0,005847953 & 0,006659 \\
\hline Desmoncus cirrhifer & 1 & 0,000811 & 0,000811 & 1 & 0,071 & 0,005847953 & 0,006659 \\
\hline Total & 1233 & 1 & 1 & 171 & 12,21 & 1 & 2 \\
\hline
\end{tabular}

$\mathrm{NI}=$ Número de individuos, $\mathrm{D}=$ Densidad, $\mathrm{DR}=$ Densidad relativa, $\mathrm{P}=$ Presencia, $\mathrm{F}:$ Frecuencia, FR: Frecuencia relativa, IVI: Índice de valor de importancia

la localidad de Angostura reflejan la dominancia de las especies $W$. quinaria, $W$. regia, $S$. exorrhiza y $G$. cuneata, que pese a no conservar el orden de dominancia siguen siendo las especies más importantes de la comunidad de palmas aun en su máximo estado de desarrollo, tal como se muestra en la Tabla 6 .

\section{ANÁLISIS DE LAESTRUCTURA DE LAS COMUNIDADES DE PALMAS DE LAS LOCALIDADES DEESTUDIO}

Ambas localidades tuvieron dominancia de los juveniles sobre los adultos y plántulas. La estructura de la población 
Tabla 5

\section{Estructura de la comunidad de palmas (plántulas, juveniles y adultos) encontradas en la localidad de Angostura, Tadó, Chocó, Colombia}

\begin{tabular}{|c|c|c|c|c|c|c|c|}
\hline Especies & NI & D & DR & SP/TA & $\mathbf{F}$ & $\mathbf{F R}$ & IVI \\
\hline Wettinia quinaria & 307 & 0,21484 & 0,2148 & 12 & 1,00 & 0,0833 & 0,2982 \\
\hline Welfia regia & 212 & 0,14836 & 0,1484 & 12 & 1,00 & 0,0833 & 0,2317 \\
\hline Geonoma cuneata & 114 & 0,07978 & 0,0798 & 9 & 0,75 & 0,0625 & 0,1423 \\
\hline Socratea exorrhiza & 105 & 0,07348 & 0,0735 & 8 & 0,67 & 0,0556 & 0,1290 \\
\hline Geonoma deversa & 90 & 0,06298 & 0,0630 & 9 & 0,75 & 0,0625 & 0,1255 \\
\hline Pholidostachys dactyloides & 85 & 0,05948 & 0,0595 & 9 & 0,75 & 0,0625 & 0,1220 \\
\hline Geonoma stricta & 73 & 0,05108 & 0,0511 & 9 & 0,75 & 0,0625 & 0,1136 \\
\hline Asterogyne martiana & 55 & 0,03849 & 0,0385 & 9 & 0,75 & 0,0625 & 0,1010 \\
\hline Bactris hondurensis & 48 & 0,03359 & 0,0336 & 8 & 0,67 & 0,0556 & 0,0891 \\
\hline Oenocarpus minor & 52 & 0,03639 & 0,0364 & 7 & 0,58 & 0,0486 & 0,0850 \\
\hline Pholidostachys pulcra & 52 & 0,03639 & 0,0364 & 7 & 0,58 & 0,0486 & 0,0850 \\
\hline Geonoma calyptroginoidea & 34 & 0,02379 & 0,0238 & 8 & 0,67 & 0,0556 & 0,0793 \\
\hline Oenocarpus bataua & 45 & 0,03149 & 0,0315 & 6 & 0,50 & 0,0417 & 0,0732 \\
\hline Euterpe precatoria & 21 & 0,0147 & 0,0147 & 6 & 0,50 & 0,0417 & 0,0564 \\
\hline Wettinia equalis & 20 & 0,014 & 0,0140 & 6 & 0,50 & 0,0417 & 0,0557 \\
\hline Iriartea deltoidea & 22 & 0,0154 & 0,0154 & 3 & 0,25 & 0,0208 & 0,0362 \\
\hline Geonoma chococola & 18 & 0,0126 & 0,0126 & 3 & 0,25 & 0,0208 & 0,0334 \\
\hline Aiphanes acaulis & 24 & 0,01679 & 0,0168 & 2 & 0,17 & 0,0139 & 0,0307 \\
\hline Attalea alleni & 14 & 0,0098 & 0,0098 & 3 & 0,25 & 0,0208 & 0,0306 \\
\hline Bactris maraja & 21 & 0,0147 & 0,0147 & 2 & 0,17 & 0,0139 & 0,0286 \\
\hline Chamaedorea $s p$ & 11 & 0,0077 & 0,0077 & 3 & 0,25 & 0,0208 & 0,0285 \\
\hline Prestoea ensiformis & 2 & 0,0014 & 0,0014 & 2 & 0,17 & 0,0139 & 0,0153 \\
\hline Synechanthus warcewizcianu & & 0,0028 & 0,0028 & 1 & 0,08 & 0,0069 & 0,0097 \\
\hline Total & 1429 & 1 & 1 & 144 & 12 & 1 & 2 \\
\hline
\end{tabular}

$\mathrm{NI}=$ Número de individuos, $\mathrm{D}=$ Densidad, $\mathrm{DR}=$ Densidad relativa, $\mathrm{P}=$ Presencia, F: Frecuencia, FR: Frecuencia relativa,

IVI: Índice de valor de importancia.

encontrada tanto en Tutunendo como en Angostura, con un bajo porcentaje de plántulas con respecto a los juveniles y adultos, podría indicar que hay problemas de reclutamiento a nivel general de la comunidad, pues lo que se esperaría para una comunidad de palmas en buen estado es que haya más plántulas que juveniles y adultos, es decir, que la estructura muestre una curva en forma de J invertida. Sin embargo, esta situación no se presenta para todas las especies: el comportamiento estructural de las poblaciones de las especies de palmas más importantes de las localidades de Tutunendo y Angostura, permite destacar que Wettinia quinaria no solo es dominante (36\% y $21 \%$ de los individuos de las palmas de Tutunendo y Angostura) y el elemento de dosel más frecuente, apareciendo en todos los transectos levantados, sino que también posee una población bien estructurada, donde la mayoría de los individuos corresponden al estado de plántula, seguido de juveniles y adultos, presentando un modelo de distribución de J-invertida típica de poblaciones sanas y en crecimiento. La situación es un poco diferente para las poblaciones de otras especies de suma importancia por sus densidades y frecuencia como Welfia regia, Socratea exorrhiza, Pholidostachys dactyloides, Iriartea deltoidea y Geonoma cuneata; es decir, que los problemas de reclutamiento de la comunidad se evidencian en los problemas de reclutamiento de estas especies.

Esta situación se puede explicar desde varios escenarios: a) Problemas de reclutamiento a nivel de la comunidad debido a la fragmentación, pues las plántulas son la clase de tamaño más vulnerable a este proceso (Scariot 1999) y b) La forma de aprovechamiento y las partes utilizadas, si tenemos en cuenta 
Bioetnia Volumen 8 No 1 (enero-junio), 2011

\section{Tabla 6 \\ Estructura de la comunidad de palmas (adultos) encontradas en la localidad de Angostura, Tadó, Chocó, Colombia}

\begin{tabular}{|c|c|c|c|c|c|c|c|}
\hline Especies & $\mathbf{N}$ & D & DR & PS/TA & $\mathbf{F}$ & $\mathbf{F R}$ & IVI \\
\hline Wettinia quinaria & 70 & 0,13 & 0,13384 & 10 & 0,8333 & 0,085 & 0,22 \\
\hline Geonoma cuneata & 47 & 0,09 & 0,08987 & 9 & 0,75 & 0,076 & 0,17 \\
\hline Welfia regia & 43 & 0,08 & 0,08222 & 8 & 0,6667 & 0,068 & 0,15 \\
\hline Geonoma deversa & 41 & 0,08 & 0,07839 & 8 & 0,6667 & 0,068 & 0,15 \\
\hline Oenocarpus minor & 42 & 0,08 & 0,08031 & 7 & 0,5833 & 0,059 & 0,14 \\
\hline Socratea exorrhiza & 40 & 0,08 & 0,07648 & 6 & 0,5 & 0,051 & 0,13 \\
\hline Geonoma stricta & 31 & 0,06 & 0,05927 & 8 & 0,6667 & 0,068 & 0,13 \\
\hline Bactris hondurensis & 24 & 0,05 & 0,04589 & 7 & 0,5833 & 0,059 & 0,11 \\
\hline Asterogyne martiana & 25 & 0,05 & 0,0478 & 7 & 0,5833 & 0,059 & 0,11 \\
\hline Pholidostachys pulcra & 26 & 0,05 & 0,04971 & 6 & 0,5 & 0,051 & 0,1 \\
\hline Oenocarpus bataua & 31 & 0,06 & 0,05927 & 5 & 0,4167 & 0,042 & 0,1 \\
\hline Geonoma calyptroginoidea & 22 & 0,04 & 0,04207 & 7 & 0,5833 & 0,059 & 0,1 \\
\hline Pholidostachys dactyloides & 15 & 0,03 & 0,02868 & 5 & 0,4167 & 0,042 & 0,07 \\
\hline Euterpe precatoria & 15 & 0,03 & 0,02868 & 5 & 0,4167 & 0,042 & 0,07 \\
\hline Wettinia equalis & 11 & 0,02 & 0,02103 & 5 & 0,4167 & 0,042 & 0,06 \\
\hline Iriartea deltoidea & 15 & 0,03 & 0,02868 & 3 & 0,25 & 0,025 & 0,05 \\
\hline Geonoma chococola & 10 & 0,02 & 0,01912 & 3 & 0,25 & 0,025 & 0,04 \\
\hline Attalea alleni & 5 & 0,01 & 0,00956 & 3 & 0,25 & 0,025 & 0,03 \\
\hline Aiphanes acaulis & 5 & 0,01 & 0,00956 & 2 & 0,1667 & 0,017 & 0,03 \\
\hline Prestoea ensiformis & 2 & 0 & 0,00382 & 2 & 0,1667 & 0,017 & 0,02 \\
\hline Synechanthus warcewizcianus & 2 & 0 & 0,00382 & 1 & 0,0833 & 0,008 & 0,01 \\
\hline Bactris maraja & 1 & 0 & 0,00191 & 1 & 0,0833 & 0,008 & 0,01 \\
\hline Chamaedorea sp. & 0 & 0 & 0 & 0 & 0 & 0 & 0 \\
\hline Total & 523 & 1 & 1 & 118 & 9,8333 & 1 & 2 \\
\hline
\end{tabular}

$\mathrm{NI}=$ Número de individuos, $\mathrm{D}=$ Densidad, $\mathrm{DR}=$ Densidad relativa, $\mathrm{P}=$ Presencia, $\mathrm{F}:$ Frecuencia, FR: Frecuencia relativa,

IVI: Índice de valor de importancia.

que para las dos localidades se registra un alto potencial de uso de tallos y frutos de individuos adultos, lo que puede influir de manera directa en la producción de semillas, el reclutamiento de las mismas y el establecimiento al interior de los bosques. Específicamente, el bajo reclutamiento que se observa para especies como Iriartea deltoidea y Socratea exorhiza muy posiblemente se relaciona con el uso de los troncos de estas especies en construcción en la zona de estudio. Para las otras especies de las que no se utilizan los troncos, el inadecuado corte de los tallos para colectar las hojas, podría ser el factor que esté provocando el bajo reclutamiento.

Es importante destacar que aunque las dos localidades presentan problemas de reclutamiento, Tutunendo tiene un mejor estado en su estructura general, más cercana al de una J- invertida, con respecto a la comunidad de palmas en Angostura. Esta situación podría estar relacionada con una mayor fragmentación del área o un estado de conservación más deficiente de Angostura en relación con Tutunendo. Aunque las respuestas específicas a esto requieren de otro tipo de estudios, es indudable que el estudio de la estructura de una comunidad se constituye en un elemento diagnóstico importante que permite inferir acerca del estado de conservación de los bosques.

Vale la pena resaltar que la situación aún no es tan dramática y todavía se encuentra una riqueza muy importante de especies y un alto número de individuos; no obstante, de continuar con los procesos de fragmentación y formas de uso, 
a largo plazo se podrían generar cambios en la composición de la comunidad de palmas ocasionados por la colonización parcial o total de especies generalistas con buena estructura poblacional como Wettinia quinaria, mientras las especies naturalmente más escasas como Aiphanes acaulis, Chamaedorea deneversiana, Geonoma chococola, Mauritiella macroclada, que necesitan ambientes más específicos y poca competencia, desaparecerían, afectando directamente la riqueza de las palmas de las localidades de estudio y a su vez la vida silvestre que aprovecha el recurso que estas ofrecen.

Las comparaciones entre las clases de tamaño de las comunidades de palmas de las localidades de estudio y otras zonas del Pacífico resultan ser difíciles, ya que los estudios florísticos generalmente no incluyen censos y datos demográficos para este grupo. Sin embargo, Scariot (1999) encontró en bosques fragmentados de Amazonía central que la clase de tamaño dominante corresponde a los juveniles (21 individuos por 0,4 ), con $36 \%$ del total de la comunidad de palmas, escenario similar al encontrado en Tutunendo y Angostura.

Las especies que dominaron el hábito arbóreo en la comunidad de palmas de las localidades de Tutunendo y Angostura fueron $W$. quinaria, $W$. regia, S. exorrhiza, $I$. deltoidea, O. bataua, O. minor y E. precatoria. Ramírez et al. (2009) reportan para los bosques inundables de las ciénagas del Medio Atrato la dominancia de las especies Euterpe precatoria, seguida de Oenocarpus batana y O. minor. Asimismo, Palacios et al. (2000), reportan a Oenocarpus bataua y Socratea exorrhiza como las especies dominantes del hábito arbóreo en los bosques del corregimiento de Salero, Chocó. Situación similar ocurre en los bosques de Nauca y Chachajo en el Alto Baudó, Chocó, según Mosquera et al. (2007). En este mismo sentido, Galeano (2001) reporta a Wettinia quinaria y Welfia regia como las palmas arbóreas más representativas en tres localidades del municipio de Nuquí, en la costa pacífica del Chocó. Rangel (2004), por su parte, afirma que en el bosque pluvial del Chocó, dominan las palmas arbóreas Welfia regia, Oenocarpus bataua y Wettinia quinaria.

\section{CONCLUSIONES}

El uso de las especies tiene en la actualidad un impacto negativo que se evidencia en la estructura de las poblaciones de las especies más aprovechadas. Tal es el caso de Welfia regia, cuya estructura de la población presenta más juveniles $(50 \%)$ y adultos (26\%) que plántulas (24\%), situación que se puede relacionar con la categoría de uso (construcción) y el órgano utilizado (tallos y hojas), pues para la cosecha es frecuente que las palmas sean derribadas, lo que explicaría la deficiencia en el reclutamiento. De igual forma, el uso impacta de forma negativa las poblaciones de otras especies importantes como Iriartea deltoidea, Socratea exorrhiza, Oenocarpus bataua, Oenocarpus minor y Geonoma cuneata.

Es importante destacar que aunque las dos localidades presentan problemas de reclutamiento, Tutunendo tiene un mejor estado en su estructura general, más cercana al de una J- invertida, con respecto a la comunidad de palmas en Angostura. Esta situación podría estar relacionada con una mayor fragmentación del área o un estado de conservación más deficiente de Angostura en relación con Tutunendo. Aunque las respuestas específicas a esto requieren de otro tipo de estudios, es indudable que el estudio de la estructura de una comunidad se constituye en un elemento diagnóstico importante que permite inferir acerca del estado de conservación de los bosques.

\section{RECOMENDACIONES}

Tomando las palmas como un recurso clave del que depende el mantenimiento de los procesos biológicos, ecológicos y evolutivos de los bosques del centro del Chocó y una parte importante de la subsistencia de sus habitantes, se requiere con urgencia reforzar las medidas de conservación en las áreas estudiadas, ampliándolas y creando áreas de monitoreo y manejo, donde se puedan conservar y usar adecuadamente estos recursos.

- Elaborar planes de manejo y modelos productivos para las especies con mayor reporte de uso en las localidades de estudio, como Iriartea deltoidea, Welfia regia, Socratea exorrhiza, Oenocarpus bataua y Oenocarpus minor.

- Teniendo en cuenta que un alto porcentaje de la comunidad de palmas en ambas localidades presenta problemas en sus estructuras poblacionales, es importante generar investigaciones puntuales para detectar y mitigar esta problemática.

- Establecer parcelas permanentes de investigación en las dos localidades, para realizar monitoreos periódicos a las especies que se relacionan con el uso y que presentan evidencia de problemas estructurales, como es el caso de Socratea exorrhiza, Iriartea deltoidea, Welfia regia, Geonoma cuneata, Pholidostachys dactyloides, Oenocarpus bataua, O, minor, Desmoncus cirrhifer y Euterpe precatoria.

\section{LITERATURACITADA}

Balick, M. J. 1982. Palmas neotropicales: nuevas fuentes de aceites comestibles. Interciencia 7 (1): 25-9.

Benz, B. F., J. Cevallos, F. Santana, J. Rosales, S. Graf. 2000. Loosing knowledge about plant use in the Sierra de Manantlan biosphere reserve, Mexico. Econ Botany 54: 183-191.

Bernal, R., G. Galeano. 1993. Palmas del andén Pacífico. p. 220-31. En: Leyva, P. (ed). Colombia Pacífico. Tomo I. Bogotá: Editorial del Fondo FEN. 


\section{Bioetnia Volumen 8 No 1 (enero-junio), 2011}

Bjorholm, S., J-C. Svenning, F. Skov, H. Balslev. 2005. Environmental and spatial controls of palm (Arecaceae) species richness across the Americas. Global Ecol Biogeogr. 14: 423-9.

Byg, A., H. Balslev. 2004. Factors affecting local knowledge of palms in Nangaritza Valley in South-Eastern Ecuador. J Etnhnobiol. 24 (2): 255 78.

Castro, A., D. Abadía, N. Pino. 2004. Plantas silvestres alimenticias de uso tradicional en las comunidades de Pacurita, San José de Purré y Guayabal, municipio de Quibdó, Chocó. Revista Institucional Universidad Tecnológica del Chocó. 18: 37-42.

Eslava, J. 1993. Climatología. pp. 136-47. En: Leyva, P. (ed). Colombia Pacífico. Tomo I. Bogotá: Editorial del Fondo FEN.

Forero, E., A. H. Gentry. 1989. Lista anotada de las plantas del departamento del Chocó, Colombia. Bogotá: Instituto de Ciencias Naturales, Museo de Historia Natural, Biblioteca José Jerónimo Triana, Universidad Natural de Colombia. $142 \mathrm{pp}$.

Galeano, G. 2001. Estructura, riqueza y composición de plantas leñosas en el golfo de Tribugá, Chocó, Colombia. Caldasia. 23 (1): 213-36.

Galeano, G., R. Bernal. 2004. Catálogo de espermatofitos en el Chocó biogeográfico familia Arecaceae. p. 135-48. En: Rangel-CH, J. O. (ed). Colombia Diversidad Biótica IV: El Chocó biogeográfico/Costa pacífica. Bogotá: Instituto de Ciencias Naturales Universidad Nacional de Colombia.

Galeano, G., R. Bernal. 2005. Palmas. p. 59-223. En: Calderón, E., G. Galeano, N. García (eds). Libro Rojo de Plantas de Colombia. Volumen II: Palmas, frailejones y zamias. Bogotá: Instituto Alexander von Humboldt, Instituto de Ciencias Naturales, Universidad Nacional de Colombia, Ministerio de Ambiente, Vivienda y Desarrollo Territorial.

García, F., J. Palacios, Y. Ramos, A. Mena, J. E. Arroyo, M. González. 2002. Composición, estructura y etnobotánica de un bosque pluvial tropical (bpT) en Salero, Chocó. Revista Institucional Universidad Tecnológica del Chocó. 17: 3-9.

Holdridge, L. R. 1996. Ecología basada en zonas de vida. San José: Instituto Interamericano de Cooperación para la Agricultura (ICCA). 225 pp.

Johnson, D. 1996. Palms: Their conservation and sustained utilization. Cambridge: IUCN. 166 pp.
Macía, M., H. Romero, R. Valencia. 2001. Patrones de uso en un bosque primario de la Amazonía ecuatoriana: comparación entre dos comunidades Huaorani. p. 225-50. En: Duivenvoorden, J., H. Balslev, J. Cavelier, C. Grandez, $\mathrm{H}$. Tuomisto, R. Valencia (eds). Evaluación de recursos vegetales no maderables en la Amazonía noroccidental. Amsterdam: IBED, Universiteit van Ámsterdam.

Mosquera, L., A. Asprilla, D. Robledo. 2007. Diversidad florística de dos zonas de bosque tropical húmedo en el municipio de Alto Baudó, Chocó, Colombia. Acta Biol Colomb. 12 (1): 75-90.

Palacios, Y., J. Lloreda., J. E. Arroyo, A. Cogollo. 2000. Composición florística de un (bp-T) en la parcela permanente de Salero, Unión Panamericana, Chocó. En: García, F., Y. Ramos, J. Palacios, J. E. Arrollo, A. Mena, M. Gonzales. (eds). Diversidad biológica de un bosque pluvial tropical. Quidbó: Universidad Tecnológica del Chocó, Instituto de Investigaciones Ambientales del Pacífico, Comunidad de Salero.

Ramírez, G., Z. Valoyes, J. Cuesta. 2009. Vegetación del bosque inundable y tierra firma del complejo cenagoso la Grande, Beté-Chocó. Bioetnia. 6(1): 16-23.

Rangel, J. O., A. Velásquez. 1997. Métodos de estudio de la vegetación. p.5987. En: Rangel, J. O., P. D. Lowey, M. Aguilar. Colombia Diversidad Biótica II. Bogotá: ICN.

Rangel-CH, O. (ed). 2004. Colombia Diversidad Biótica IV: El Chocó biogeográfico/costa pacífica. Bogotá, DC: Instituto de Ciencias Naturales Universidad Nacional de Colombia.1024 pp.

Scariot, A. O. 1999. Forest fragmentation effects on palm diversity in central Amazon. J Ecol. 87: 66-76.

Valois, H., N. Pino. 2004. Ethnobotany of four black communities of the municipality of Quibdó, Chocó, Colombia. Etnobotánica de cuatro comunidades negras del municipio de Quibdó, Chocó, Colombia. Lyonia J Ecol Applic. 7 (2): 61-9.

Villareal, H., M. Álvarez, S. Córdoba, F. Escobar, G. Fagua, F. Gast, et al. 2004. Manual de métodos para el desarrollo de inventarios de biodiversidad. Programa de Inventarios de Biodiversidad. Bogotá, DC: Instituto de Investigación de Recursos Biológicos Alexander Von Humboldt. p. 1-236. 